\title{
The secreted protein acidic and rich in cysteine (SPARC) induces endoplasmic reticulum stress leading to autophagy-mediated apoptosis in neuroblastoma
}

\author{
G.S. SAILAJA ${ }^{1}$, PRAVEEN BHOOPATHI ${ }^{1}$, BHARATHI GORANTLA ${ }^{1}$, CHANDRAMU CHETTY $^{1}$, \\ VENKATESWARA RAO GOGINENI ${ }^{1}$, KIRAN KUMAR VELPULA ${ }^{1}$, CHRISTOPHER S. GONDI $^{1}$ and JASTI S. RAO ${ }^{1,2}$ \\ Departments of ${ }^{1}$ Cancer Biology and Pharmacology, and ${ }^{2}$ Neurosurgery, \\ University of Illinois College of Medicine at Peoria, Peoria, IL 61605, USA
}

Received August 13, 2012; Accepted September 21, 2012

DOI: $10.3892 /$ ijo.2012.1678

\begin{abstract}
Our previous studies showed that overexpression of secreted protein acidic and rich in cysteine (SPARC) induced autophagy-mediated apoptosis in PNET cells. In the present study, we attempted to elucidate the molecular mechanisms and signaling cascades associated with SPARC overexpression in combination with radiation therapy that eventually leads to autophagy-mediated apoptosis in neuroblastoma. SPARC expression in SK-N-AS and NB-1691 cells demonstrated the activation of caspase 3 , cleavage of PARP and induction of apoptosis. The experiments to unravel the mechanisms associated with autophagy-apoptosis illustrated that SPARC overexpression triggered endoplasmic reticulum (ER) stress and thereby unfolded protein response (UPR). This was apparent with the activation of stress receptors, inositol-requiring enzyme (IRE $1 \alpha$ ), RNA-dependent protein kinase (PKR)-like ER kinase (PERK) and BiP. This study further demonstrated the induction of transcription factor CHOP as a result of IRE-JNK activation in response to increased SPARC levels. Inhibition of ER stress and JNK activation led to inhibition of autophagy-mediated apoptosis. Further, the apparent expression of ER stress molecules among the orthotopic tumors treated by SPARC overexpression plasmids substantiated our in vitro observations. Taken together, these results illustrate the critical role of ER stress in regulating autophagy-mediated apoptosis in SPARC-overexpressed neuroblastoma cells and radiation treatment.
\end{abstract}

\section{Introduction}

Neuroblastoma, the most common extracranial pediatric malignancy, is characterized by a broad spectrum of clinical behaviors

Correspondence to: Dr Jasti S. Rao, Department of Cancer Biology and Pharmacology, University of Illinois College of Medicine, One Illini Drive, Peoria, IL 61605, USA

E-mail: jsrao@uic.edu

Key words: secreted protein acidic and rich in cysteine, neuroblastoma, autophagy, apoptosis, endoplasmic reticulum stress with a low survival rate $(1,2)$. The clinical prognosis reveals its aggressive behavior, including wide dissemination, resistance to chemotheraphy, and ability to metastasize to the bone (3). Since the tumor is associated with a high fatality rate, various investigators are working to improve the survival rate, reduce recurrence, and metastasis of neuroblastoma. Like any other malignant tumor, the hallmarks of high-risk neuroblastoma are unregulated cellular proliferation and declined apoptosis. Apoptosis is the intrinsically programmed cell death that is necessary to maintain normal homeostasis of the system. Its diminution violates many of the physiological checkpoints inside the cell and elicits abnormal behavior in the cellular microenvironment. The potentially detrimental effects observed with the absence/reduction of apoptosis can be considered as a function of the activation of anti-apoptotic signaling cascades (4). Given the important role of apoptosis regulators in neuroblastoma, a better understanding of the underlying mechanisms involved in the induction of apoptosis would be a very important aspect in designing anticancer drugs and developing efficient therapeutic strategies.

Secreted protein acidic and rich in cysteine (SPARC) is an evolutionary matricellular glycoprotein composed of three structural domains with distinct modular functions and plays critical roles in modulating cell-matrix interactions, cellular functions and tissue mineralization (5). SPARC plays a significant role in tissue remodeling, embryogenesis, cellular differentiation and angiogenesis. As a matricellular protein, SPARC takes part in multiple biological activities including development and regulation of matrix remodeling. As such, molecular mechanisms associated with variation in SPARC levels in the cell would have decisive consequences in regulating the diverse cellular functions.

The underlying mechanisms involved in SPARC expression and malignant tumor regression remain elusive; however, it is known that SPARC expression is highly dependent on various factors including tumorigenic phenotype, molecular signaling pathways associated with integrins and growth factors/chemokines (5). Even though the comprehensive role of SPARC-mediated tumor regression is not completely understood, previous studies have clarified the anti-proliferative and counter adhesive properties of SPARC through specific cytokines and growth factors (6-8). 
The significance of combination therapy arises from the possibility of achieving a synergistic effect in advanced treatment modalities. Different combination therapies, like combination of chemotherapy and radiation therapy, have been investigated to improve the efficiency (9). Radiation has been recognized as an efficient mode of therapy for neuroblastoma and provides an additive pharmacological response when combined with other treatments $(10,11)$.

Our recent findings showed SPARC overexpression inhibited cell proliferation, migration and angiogenesis in PNET cells $(12,13)$. Earlier reports from our group also proved that SPARC overexpression induced autophagy-mediated apoptosis in PNET cells (14). However, the molecular mechanisms associated with SPARC overexpression leading to autophagy-mediated apoptosis have not been studied. In the present study, we attempted to elucidate the efficacy of SPARC overexpression together with radiation therapy as an efficient way to induce apoptosis in neuroblastoma as well as investigate associated molecular mechanisms.

This study provides solid evidence describing the key role of endoplasmic reticulum (ER) stress in invoking the transcriptional responses as a function of SPARC overexpression. ER is the sole element responsible for protein synthesis and folding; an imbalance between the cellular demand and the capacity of ER to facilitate protein folding eventually activates aberrant cell cycle regulation, deregulates the intercellular metabolism, and eventually activates ER stress molecular chaperons. Our study was also focused on understanding the specific pathways associated with SPARC overexpression that induce ER stress, which in turn elicit autophagy-mediated apoptosis in neuroblastoma. The induction of autophagy as a result of ER stress has been identified by various investigators $(15,16)$. Autophagy involves sequestration of autophagosomes that eventually fuse with lysosomes and lead to cellular degradation; as a result, autophagy has an important role in eukaryotic cells. Hence, we also investigated the involvement of autophagy as an event associated with SPARC overexpression and induction of apoptosis in neuroblastoma.

\section{Materials and methods}

Cell culture. SK-N-AS and NB-1691 cells were procured from ATCC (Manassas, VA) and Dr P. Houghton of St. Jude Children's Research Hospital (Memphis, TN), respectively. The cells were maintained in RPMI-1640 supplemented with 10\% FBS (Invitrogen Corp., Carlsbad, CA), $50 \mathrm{U} / \mathrm{ml}$ penicillin and $50 \mu \mathrm{g} /$ $\mathrm{ml}$ streptomycin (Life Technologies, Inc., Frederick, MD). Cells were maintained in a $37^{\circ} \mathrm{C}$ incubator with a $5 \% \mathrm{CO}_{2}$ humidified atmosphere.

Antibodies and reagents. The primary antibodies against SPARC, caspase 3, LC3, JNK, phospho-JNK, pancreatic ER kinase (PERK), GAPDH (Santa Cruz Biotechnology, Santa Cruz, CA), PARP, inositol-requiring enzyme $1 \alpha$ (IRE- $1 \alpha)$, BiP, and CHOP (Cell Signaling Technology, Beverly, MA) were used in this study. HRP- or Alexa Fluor-488/Alexa Fluor-594conjugated secondary antibodies, isotype control IgG (Santa Cruz Biotechnology, Santa Cruz, CA), Vectashield mounting medium with DAPI (Vector Laboratories, Burlingame, CA), DAB peroxidase substrate (Sigma, St. Louis, MO), TUNEL (terminal deoxynucleotidyl transferase-mediated dUTP nickend-labeling) detection kit (Roche Molecular Biochemicals, Indianapolis, IN), JNK Inhibitor II SP600125 (Calbiochem, San Diego, CA), and Salubrinal (ER stress inhibitor, Fisher) were also used in this study.

Construction of pcDNA3.1-SPARC, transfection and irradiation of neuroblastoma cells. Human SPARC cDNA was amplified by PCR using synthetic primers and was cloned into a pcDNA3.1 vector (Invitrogen, San Diego, CA) in sense orientation as described previously (17). Neuroblastoma cells were transfected with plasmid vector containing full-length cDNA of SPARC (pSPARC) or empty vector (pEV) using FuGene HD (Roche, Indianapolis, IN) as described earlier (18). After 4-6 h of transfection, the necessary amount of serum-containing medium was added. After $24 \mathrm{~h}$ of incubation, cells were irradiated with X-ray irradiation at a dose of 8 Gy using the RS 2000 Biological Irradiator (Rad Source Technologies, Inc., Boca Raton, FL). Then, the medium replaced, and cells were incubated for a further $16 \mathrm{~h}$ or for the indicated time period.

Immunocytochemistry. Immunocytochemistry was performed as described previously (17). Briefly, cells were cultured on 8-well chamber slides and transfected as above. Forty-eight hours after transfection, cells were fixed with $4 \%$ freshly prepared paraformaldehyde (w/v) in PBS followed by permeabilization with $0.1 \%$ Triton X-100 (w/v) in PBS and blocked with $1 \%$ BSA (w/v) in PBS for $1 \mathrm{~h}$ at $4^{\circ} \mathrm{C}$. Cells were incubated overnight at $4^{\circ} \mathrm{C}$ with anti-SPARC or anti-CHOP antibody followed by corresponding Alexa Fluor-488- or Alexa Fluor-594-conjugated secondary antibody for $1 \mathrm{~h}$ at room temperature. Slides were mounted with Vectashield mounting medium with DAPI (Vector Laboratories) and analyzed under a microscope (Olympus BX61 Fluoview, Minneapolis, MN). Isotype control IgG served as a negative control.

Western blotting. Western blot analysis was performed as reported earlier (19). Briefly, $48 \mathrm{~h}$ after transfection, cells were collected and lysed in RIPA buffer. Equal amounts of protein were resolved on SDS-PAGE and transferred onto a PVDF membrane. The blots were blocked with $5 \%$ non-fat dry milk and probed overnight with primary antibodies followed by HRP-conjugated secondary antibodies. ECL system was used to detect chemiluminescent signals. All blots were re-probed with GAPDH antibody to confirm equal loading.

Reverse transcription polymerase chain reaction (RT-PCR). Neuroblastoma cells were transfected with mock, pEV or pSPARC for $36 \mathrm{~h}$. Total RNA was extracted from these cells and cDNA synthesized using poly-dT primers as described earlier (20). PCR was performed using the following primers: SPARC: 5'-GGAAGAAACTGTGGCAGAGG-3' (sense), and 5'-ATTGCTGCACACCTTCTCAA-3' (antisense); GAPDH: 5'-TGAAGGTCGGAGTCAACGGATTTGGT-3' (sense), and 5'-CATGTGGGCCATGAGGTCCACCAC-3' (antisense).

Flow cytometry. FACS analysis was used to assess cell cycle phases (FACSCalibur System, BD Bioscience, San Jose, CA) with laser excitation at $488 \mathrm{~nm}$ and an emission at $639 \mathrm{~nm}$ band pass filter to collect red propidium iodide fluorescence. 
A

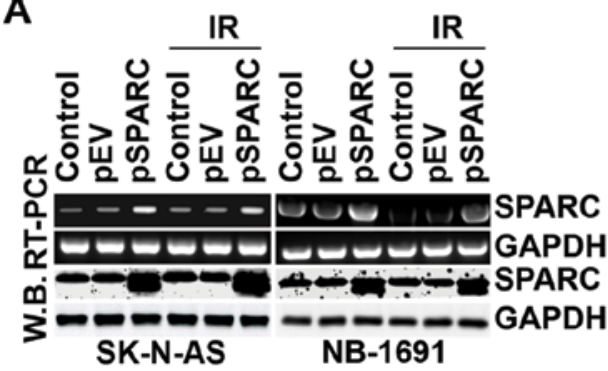

B
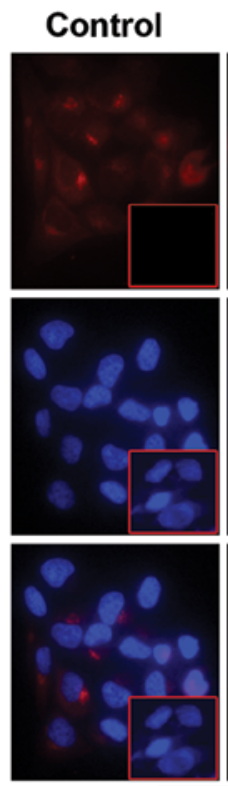

pEV
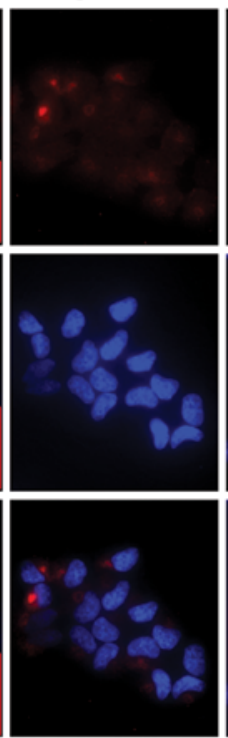

SK-N-AS

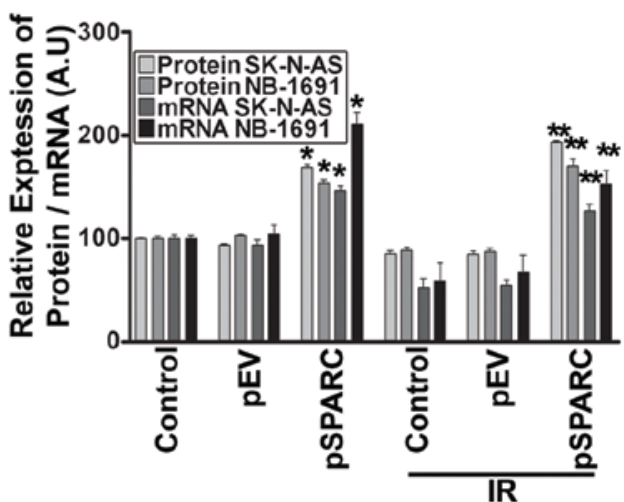

PSPARC
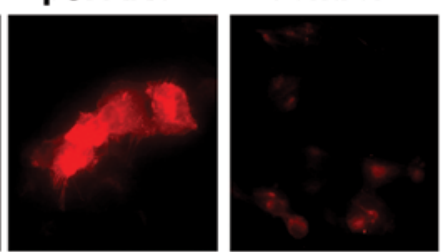

pEV

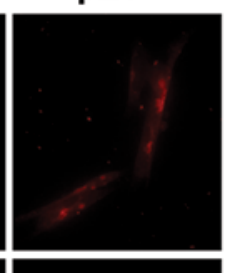

pSPARC
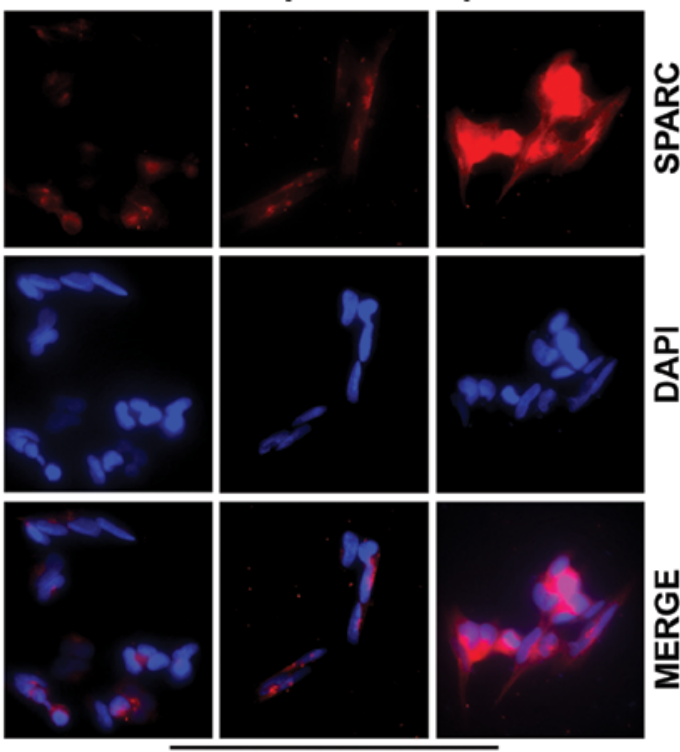

NB-1691

Figure 1. SPARC overexpression in neuroblastoma cells. (A) SK-N-AS and NB-1691 neuroblastoma cells were seeded in dishes and left overnight. Cells were transfected with pEV or pSPARC and cultured. After $24 \mathrm{~h}$, cells were irradiated with 8 Gy and incubated for another $16 \mathrm{~h}$. Total RNA was extracted from the control, transfected and irradiated cells. RT-PCR was performed for SPARC using specific primers. GAPDH served as a control. SPARC levels were determined by western blot analysis using a SPARC-specific antibody. GAPDH served as a loading control. Results are representative of three independent experiments. Densitometric analysis showing levels of SPARC protein and mRNA levels. Columns, mean of three experiments; bar, \pm SD. * $<<0.01$ vs. pEV; ${ }^{* *}$ p $<0.01$ vs. IR + pEV. (B) Neuroblastoma cells were cultured in 8-well chamber slides and transfected with pEV or pSPARC. After 36 h, cells were fixed, blocked and incubated overnight with anti-SPARC primary antibody followed by incubation with Alexa Fluor-594-conjugated secondary antibody for 1 h. Slides were mounted with Vectashield mounting medium containing DAPI and photographed under a confocal microscope. Inset, negative control with isotype control IgG.

The percentages of cells in the various phases of the cell cycle (sub-G0/G1, S, and G2/M) were assessed using Cell Quest software (BD Bioscience, San Jose, CA).

Terminal deoxy nucleotidyl transferase-mediated nick labeling (TUNEL) assay and immunohistochemistry. Apoptosis in neuroblastoma cells after SPARC transfection alone and in combination was detected using TUNEL enzyme reagent according to the manufacturer's instructions and as described previously (17). Briefly, $2 \times 10^{3}$ cells were cultured in 4-well chamber slides, transfected with pSPARC, irradiated after $24 \mathrm{~h}$ of transfection, fixed in $4 \%$ paraformaldehyde in PBS for $1 \mathrm{~h}$ at room temperature, and permeabilized in $0.1 \%$ Triton X-100 in $0.1 \%$ sodium citrate in PBS for $10 \mathrm{~min}$ on ice. The samples were incubated in TUNEL reaction mixture in a humidified atmosphere at $37^{\circ} \mathrm{C}$ for $1 \mathrm{~h}$ in the dark. Slides were mounted and images were captured with an Olympus BX 60 research fluorescence microscope attached to a
CCD camera, and cells were counted. The apoptotic index was defined as follows: apoptotic index $(\%)=100 \mathrm{x}$ (apoptotic cells/ total cells).

Intra-adrenal tumor model and immunohistochemistry. The Institutional Animal Care and Use Committee at the University of Illinois College of Medicine at Peoria approved all experimental procedures involving the use of animals. Orthotopic, localized neuroblastoma tumors were established in C.B-17 SCID mice by injection of $1 \times 10^{6}$ NB-1691 cells in $100 \mu 1$ PBS into the retroperitoneal space as described earlier (21). After 2 weeks of tumor cell implantation, the mice were separated into six groups containing 6 animals per group, and each group was injected intravenously with PBS (mock), pEV or pSPARC (100 $\mu$ l volume) and were given three doses on alternate days. Between the first and the second injections, and the second and the third injections, one group was irradiated with 
a dose of 5 Gy each time. Mice were euthanized when they lost $>20 \%$ of body weight or had trouble ambulating, feeding, or grooming. The tumors were removed and either fixed in $10 \%$ phosphate-buffered formaldehyde or snap frozen and maintained at $-70^{\circ} \mathrm{C}$ until sectioning. Briefly, all tumors were serially sectioned and tissue sections $(5-\mu \mathrm{m}$ thick) obtained from the paraffin blocks were stained with hematoxylin and eosin (H\&E) using standard histologic techniques. For immunohistochemical analysis, sections were incubated with primary antibody for $2 \mathrm{~h}$ at room temperature followed by the HRP-conjugated secondary antibody. DAB solution was used as the chromogen. Isotype control IgG was used as a negative control. The nucleus was counterstained with hematoxylin and sections were mounted and analyzed.

Statistical analysis. All data are presented as means \pm standard error (SE) of at least three independent experiments, each performed at least in triplicate. One way analysis of variance (ANOVA) combined with the Tukey post hoc test of means was used for multiple comparisons in cell culture experiments. Statistical differences are presented at probability levels of $\mathrm{p}<0.05, \mathrm{p}<0.01$ and $\mathrm{p}<0.001$.

\section{Results}

SPARC overexpression followed by radiation therapy enhanced apoptosis in neuroblastoma cells. It has been demonstrated that SPARC overexpression induces apoptosis in PNET cells (14). In this study, initially, the role of SPARC overexpression to induce apoptosis by itself and in combination with radiation was investigated in neuroblastoma cells. The expression of SPARC significantly increased at both protein and mRNA levels in cells transfected with pSPARC (Fig. 1A). The expression was increased by $>75 \%$ in both cell lines with and without radiation combination when compared to the respective control or empty vector-treated counterparts (Fig. 1A). SPARC expression levels after transfection were also checked by immunofluorescence analysis, which also demonstrated an apparent increase in cellular expression of SPARC in pSPARC-transfected cells (Fig. 1B). Further, flow cytometric analysis showed that SPARC transfection alone or in combination with radiation (IR) dosage of 8 Gy resulted in a significant increase of the sub-G0/G1 population of cells, which indicates the induction of apoptosis in the SK-N-AS and NB-1691 neuroblastoma cells (Fig. 2A). SPARC and IR-induced apoptosis was further confirmed by TUNEL assay (Fig. 2B) and cleavage of caspase 3 and PARP (Fig. 2C). These results demonstrate that SPARC overexpression increased the sensitivity of neuroblastoma cells to radiation.

SPARC overexpression induces autophagy. Our earlier studies showed that SPARC overexpression led to autophagy-mediated apoptosis in PNET cells (14). To understand the molecular pathways associated with SPARC overexpression leading to autophagy, expression of autophagy marker protein microtubuleassociated protein light chain-3 II (LC3-II), which is formed as a result of phosphoatidylethanol-amine conjugation of LC3-I was chosen (22). Increased expression of LC3 was observed for SPARC-overexpressed neuroblastoma cell lines, which confirms autophagy as a part of the molecular events leading to apoptosis (Fig. 2D). To better understand the cellular pathways associated with SPARC overexpression leading to autophagymediated apoptosis, the role of endoplasmic reticulum (ER) stress (22) was investigated. IRE 1, a type 1 ER transmembrane bifunctional glycoprotein having serine/threonine kinase and endoribonuclease activities in its cytosolic domain, was found to be upregulated with SPARC overexpression (Fig. 2D). In addition, other ER molecular chaperons, BiP and PERK, were also found to be activated with increased SPARC expression at an early time period of $24 \mathrm{~h}$ in NB-1691 and SK-N-AS cell lines (Fig. 2D). The prolonged stress led to the upregulation of the proapoptotic transcription factor, $\mathrm{CHOP}$, which was induced as a result of DNA damage and/or other stress conditions (Fig. 2D). A significant increase of these molecules in pSPARC treated cells indicated a direct involvement of autophagy in SPARC induced apoptosis in neuroblastoma.

Endoplasmic reticulum stress activates the c-Jun $N$-terminal kinase $(J N K)$ pathway. There is a wealth of knowledge pointing out the profound role of c-Jun N-terminal kinase (JNK) in apoptosis (23). In addition, it was shown that IRE1 $\alpha$ can also initiate cell death through the activation of the JNK pathway (24). Hence, we sought to determine the effect of SPARC overexpression on phosphorylation of JNK. The results show that phospho-JNK levels increased 2 -fold as a result of SPARC overexpression (Fig. 3A). To understand the involvement of JNK pathway and ER stress molecules in the apoptosis signaling cascade, SPARCtransfected neuroblastoma cells were treated with a JNK inhibitor after radiation. When phosphorylation of JNK was inhibited by the pharmacological inhibitor, the expression levels of ER stress molecules BiP and PERK were downregulated; these results illustrate that ER stress activation was initiated by the phosphorylation of JNK (Fig. 3B). It was also found that when activation of JNK was inhibited, the TUNEL positivity of pSPARC-transfected cells either alone or in combination with radiation was significantly diminished (Fig. 3C). Further, inhibition of JNK activity resulted in a marked decrease of cleavage of caspase 3 and PARP among pSPARC-transfected cells, thereby confirming the active involvement of JNK in regulating apoptosis in these cells (Fig. 3D).

Endoplasmic reticulum stress regulates apoptosis. Next, the contributory role of ER stress to induce apoptosis as a result of JNK activation by SPARC overexpression was tested using an ER stress inhibitor in pSPARC-transfected and irradiated neuroblastoma cells. As anticipated, inhibition of ER by a pharmacological inhibitor also significantly reduced the TUNEL positivity of SK-N-AS and NB-1691 neuroblastoma cells (Fig. 4A). Further, we also noted a sharp decrease in the activation of caspase 3 and cleavage of PARP among the pSPARC-transfected and ER inhibitor-treated cells (Fig. 4B).

SPARC overexpression in combination with irradiation in an orthotopic neuroblastoma model suppresses tumor growth in vivo. Based on the in vitro results, it could be proposed that SPARC overexpression invokes ER stress, which in turn enhances autophagy-mediated apoptosis in neuroblastoma. This hypothesis was tested by orthotopically implanting NB-1691 neuroblastoma cells in mice and treating with pSPARC alone and in combination with radiation. Increased SPARC expression levels were observed in pSPARC-treated tumors 

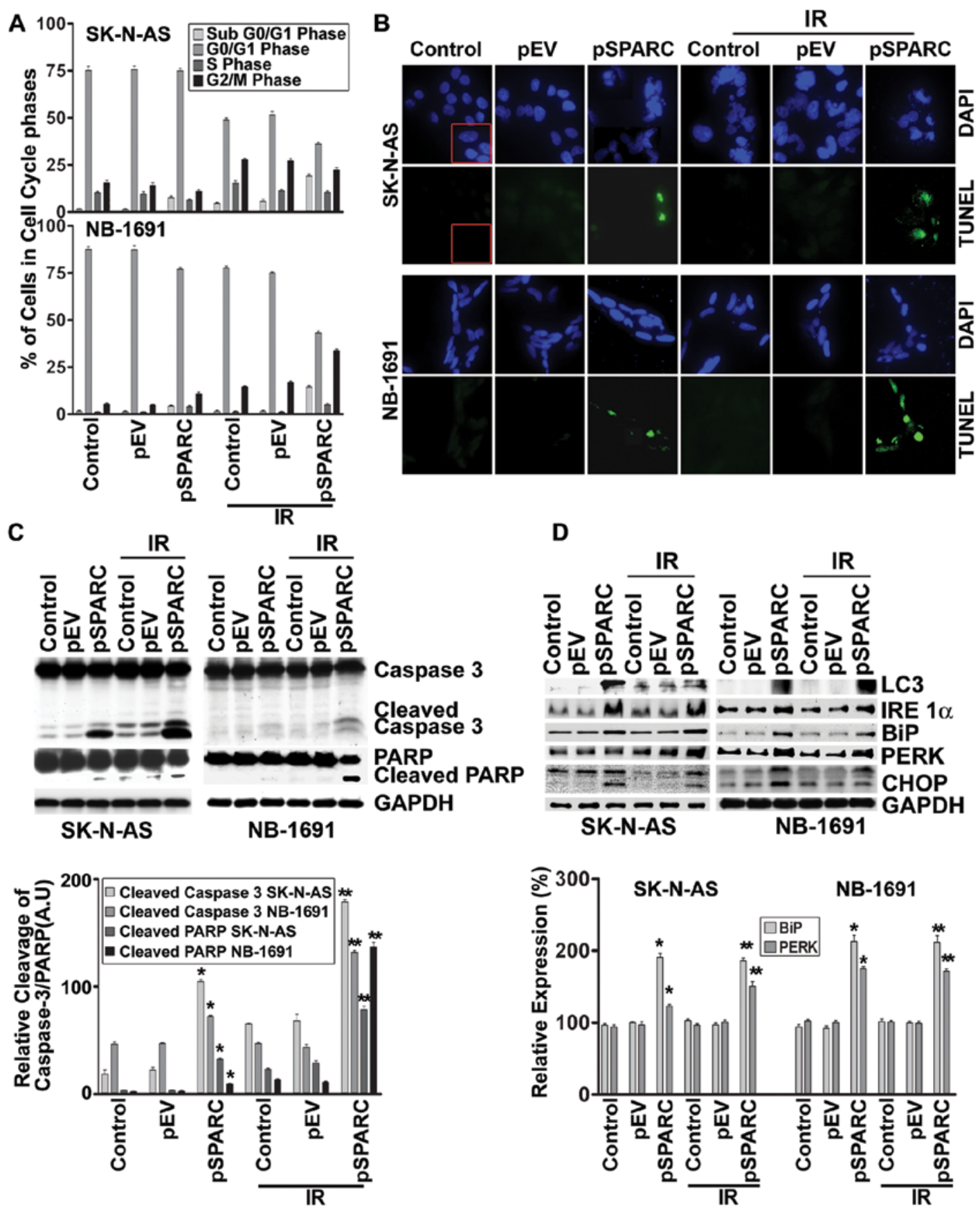

Figure 2. SPARC overexpression sensitizes cells to radiation in neuroblastoma cells. SK-N-AS and NB-1691 neuroblastoma cells were seeded in dishes and left overnight. Cells were transfected with pEV or pSPARC and cultured. After $24 \mathrm{~h}$, cells were irradiated with $8 \mathrm{~Gy}$ and incubated for another $16 \mathrm{~h}$. (A) Cells were collected and subjected to FACS analysis with propidium iodide staining for DNA content analysis and are represented in a graphical manner. Columns:

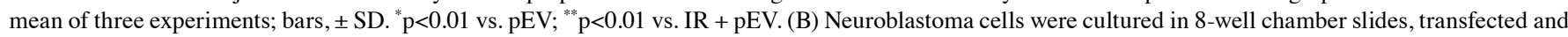
irradiated as described above. Cells were fixed with paraformaldehyde, permeabilized and blocked. Cells were incubated with TUNEL reaction mixture for $1 \mathrm{~h}$. Nuclei were counterstained with DAPI and slides were mounted and photographed. Inset, negative control. (C) Western blot analysis was performed for caspase 3 and PARP from total cell lysates using specific antibodies. GAPDH served as a loading control. Densitometric analysis showing levels of cleaved caspase 3 and PARP. Columns, mean of three experiments; bars, \pm SD. ${ }^{*} p<0.01 \mathrm{vs.} \mathrm{pEV;}{ }^{* *} p<0.01 \mathrm{vs}$. IR+pEV. (D) Total cell lysates were used for western blot analysis to detect protein levels for LC3, IRE 1 $\alpha$, BiP, PERK and CHOP using specific antibodies. GAPDH served as a loading control. Densitometric analysis showing levels of BiP and PERK. Columns: mean of three experiments; bars, \pm SD. ${ }^{*} p<0.01$ vs. pEV; ${ }^{* *} p<0.01$ vs. IR + pEV.

as compared to mock or pEV-treated tumors. The expression levels of phospho-JNK and LC3 were found to increase in tumors treated with pSPARC alone and in combination with radiation (Fig. 5A). Further, the ER stress molecules IRE $1 \alpha$, BiP, PERK and CHOP were also expressed in elevated levels in pSPARC-treated tumors (Fig. 5B). TUNEL analysis confirmed
pSPARC-induced apoptosis in vivo and a remarkable increase in apoptosis was observed with the combination treatment of pSPARC and radiation (Fig. 5C). The in vivo results thus corroborate the in vitro findings and support the hypothesis that ER stress plays a key role in regulating the induction of apoptosis in SPARC-overexpressed neuroblastoma cells. 
A

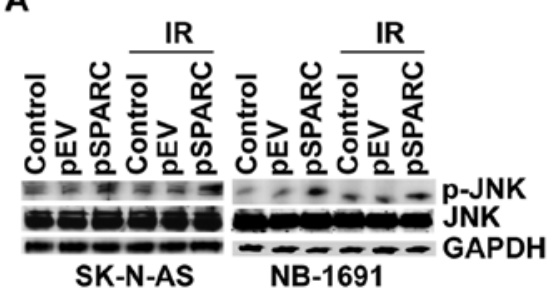

B

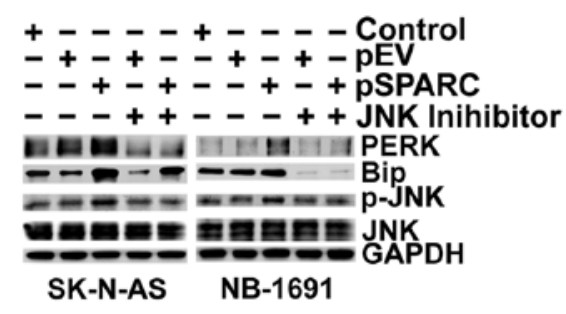

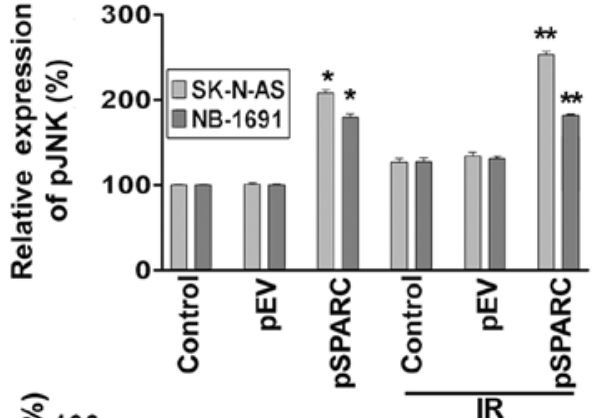

C

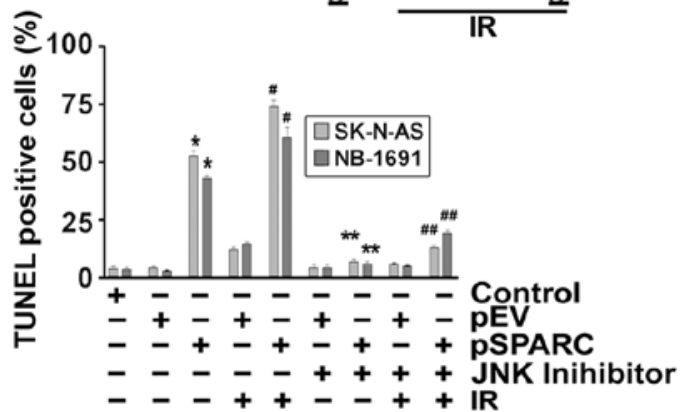

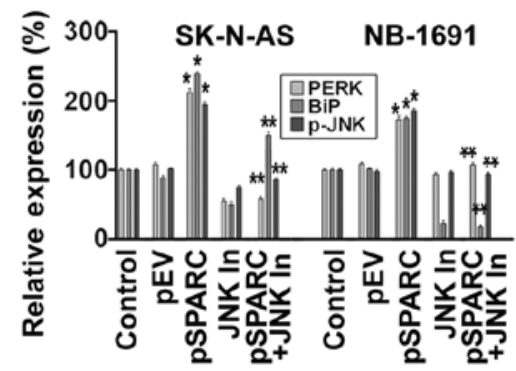

D

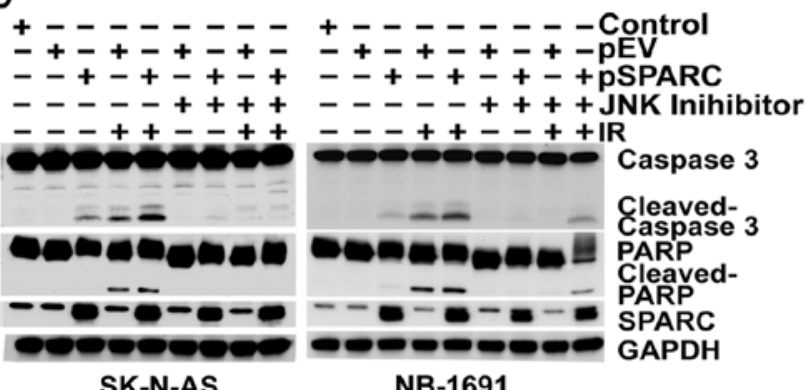

SK-N-AS

NB-1691

Figure 3. SPARC overexpression induces phosphorylation of JNK through endoplasmic reticulum (ER) stress. (A) SK-N-AS and NB-1691 neuroblastoma cells were seeded in dishes and left overnight. Cells were transfected with pEV or pSPARC and cultured. After $24 \mathrm{~h}$, cells were irradiated with 8 Gy and incubated for another $16 \mathrm{~h}$. Cells were collected and the cell lysates were subjected to western blotting for phospho-JNK (p-JNK) and JNK. GAPDH served as a loading control. Results are representative of three independent experiments. Phospho-JNK band intensities were quantified by densitometry using ImageJ (NIH) software and shown as the bar graph. Columns, mean of triplicate experiments; bars, \pm SD. ${ }^{*}$ p $<0.01$ vs. pEV; *" $p<0.01$ vs. IR + pEV. (B) Neuroblastoma cells were transfected with $p E V$ or pSPARC and cultured. After $24 \mathrm{~h}$, cells were treated with JNK inhibitor for $16 \mathrm{~h}$. Cells were collected and the lysates were subjected to western blotting for PERK, BiP, phospho-JNK (p-JNK) and JNK. GAPDH served as a loading control. Results are representative of three independent experiments. Densitometric analysis for PERK, BiP and p-JNK was performed using ImageJ (NIH) software and shown as the bar graph. Columns, mean of triplicate experiments; bars, \pm SD. ${ }^{*}<<0.01 \mathrm{vs.}$ $\mathrm{pEV} ;{ }^{* * *} \mathrm{p}<0.01$ vs. pSPARC. (C) Neuroblastoma cells were cultured in 8-well chamber slides and transfected and irradiated as described above. After irradiation, cells were treated with JNK inhibitor for $16 \mathrm{~h}$. TUNEL assay was performed as described in Fig. 2B. The TUNEL-positive cell population was quantified and shown

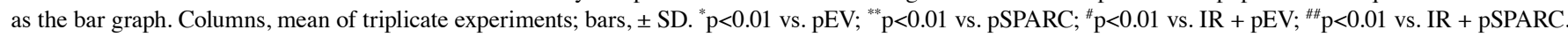
(D) Neuroblastoma cells were plated, transfected, irradiated and treated with JNK inhibitor as described above. Cells were collected and the cell lysates were subjected to western blotting for SPARC, caspase 3 and PARP. GAPDH served as a loading control. Results are representative of three independent experiments.
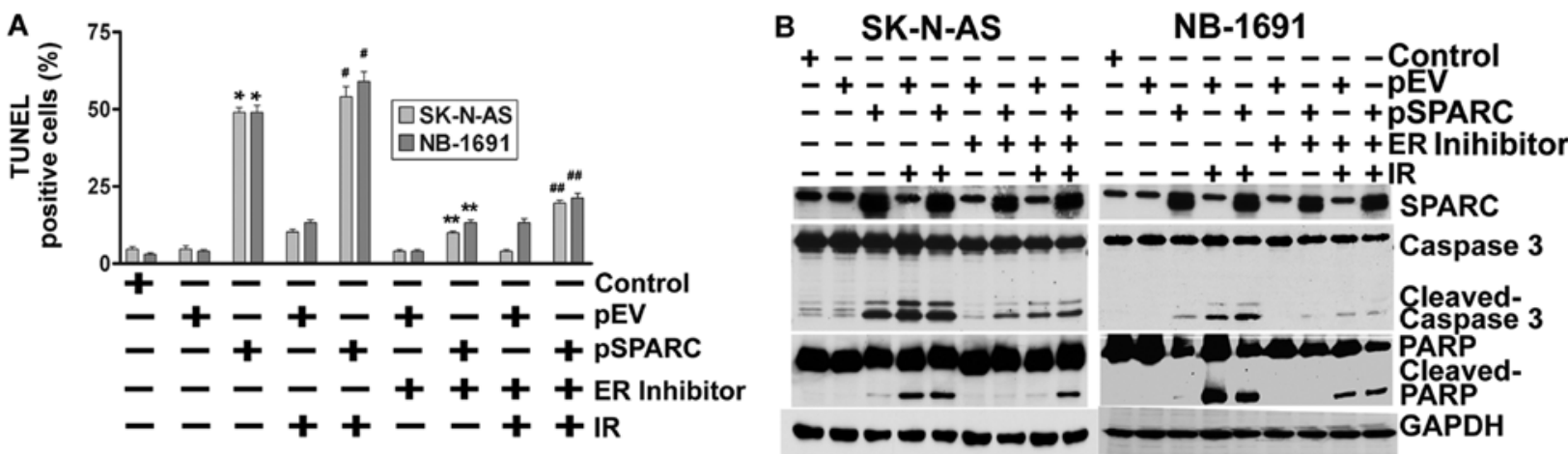

Figure 4. Endoplasmic reticulum (ER) stress regulates apoptosis. (A) SK-N-AS and NB-1691 neuroblastoma cells were seeded in 8-well chamber slides and left overnight. Cells were transfected with pEV or pSPARC and cultured. After $24 \mathrm{~h}$, cells were irradiated with 8 Gy followed by an ER inhibitor and incubated for another $16 \mathrm{~h}$. TUNEL assay was performed as described in Fig. 2B. The TUNEL-positive cell population was quantified and shown as the bar graph. Columns, mean of triplicate experiments; bars, \pm SD. ${ }^{*} \mathrm{p}<0.01$ vs. $\mathrm{pEV} ;{ }^{*} \mathrm{p}<0.01$ vs. pSPARC; ${ }^{\#} \mathrm{p}<0.01$ vs. IR + pEV; ${ }^{* \#} \mathrm{p}<0.01$ vs. IR + pSPARC. (B) Neuroblastoma cells were plated, transfected, irradiated and treated with ER inhibitor as described above. Cells were collected and the cell lysates were subjected to western blotting for SPARC, caspase 3 and PARP. GAPDH served as a loading control. Results are representative of three independent experiments. 
A

Control

pEV

PSPARC

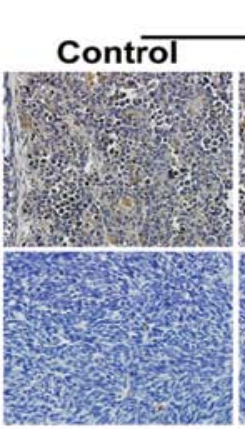

IR PSPARC
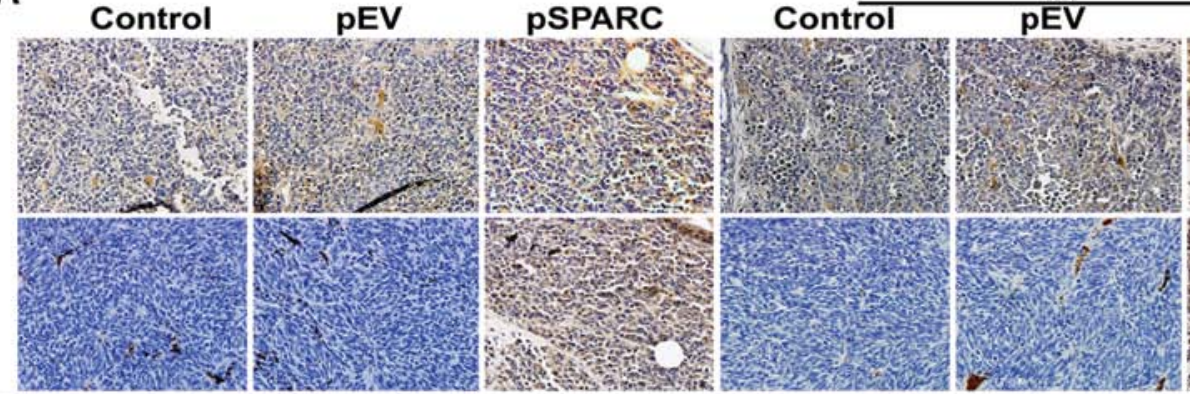

PSPARC

B
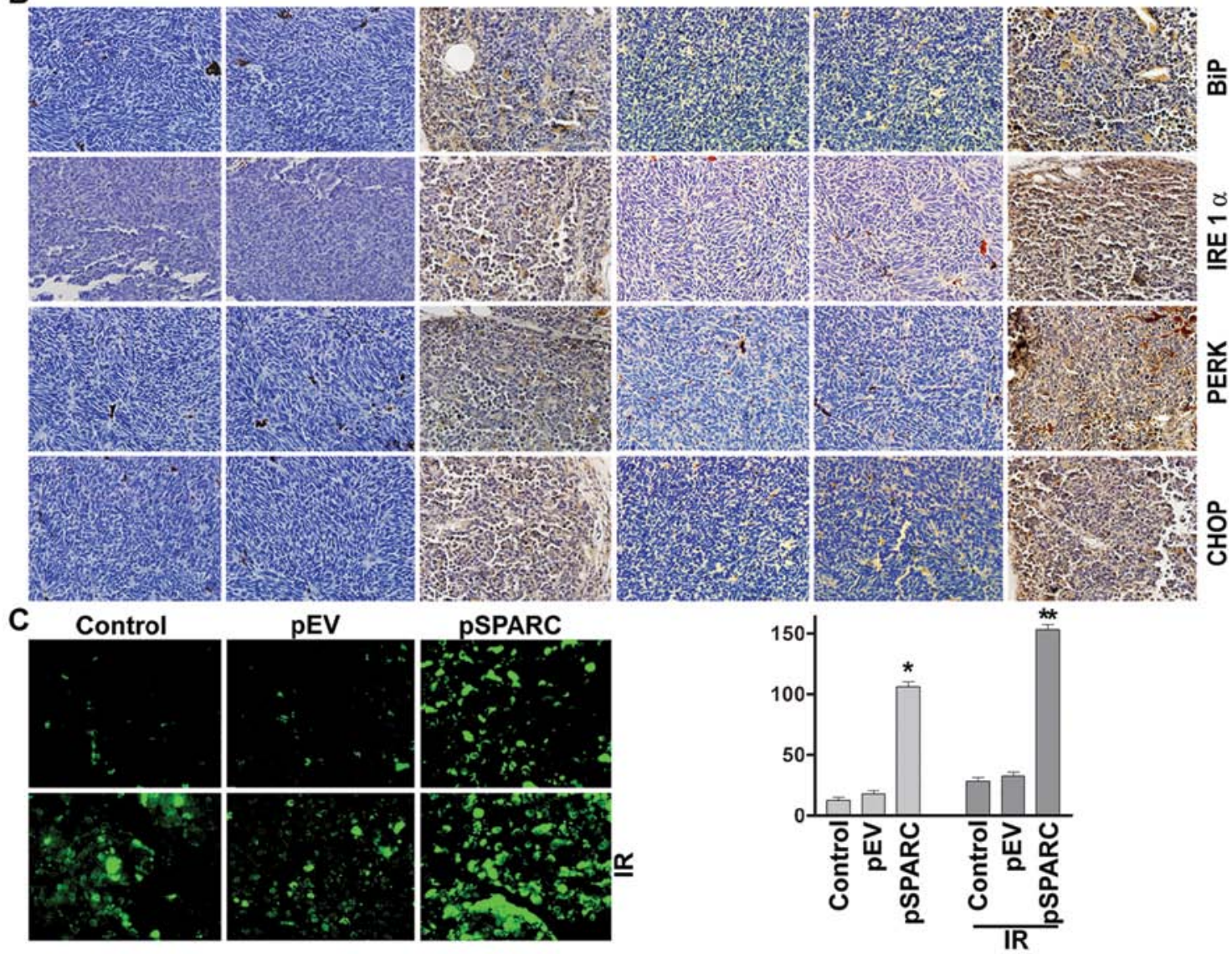

Figure 5. SPARC overexpression alone and in combination with radiation inhibits pre-established tumor growth in vivo. Neuroblastoma orthotopic tumor sections from mice injected with mock, pEV or pSPARC plasmids alone or in combination with radiation (IR) were analyzed as described in Materials and methods. (A) Immunohistochemical analysis for phospho-JNK (pJNK) and LC3 were carried out as described in Materials and methods. (B) Immunohistochemical analysis for BiP, IRE 1 $\alpha$, PERK and CHOP were carried out as described in Materials and methods. All results are representative of multiple tumors taken from five separate mice in each treatment group (magnification x60). Inset, negative control. (C) Staining for TUNEL-positive cells in xenograft tissue sections from mock, pEV, and pSPARC alone or in combination with radiation ( 2 doses of $5 \mathrm{~Gy}$ )-treated mice was performed as described in Materials and methods. TUNEL-positive cells were quantified and shown as bar graph. Columns, mean of triplicate experiments; bars, \pm SD. ${ }^{*}$ p $<0.01$ vs. pEV; ${ }^{* *}$ p $<0.01$ vs. IR + pEV.

In addition, these results demonstrate the involvement of LC3 and active JNK as part of the signal pathway leading to apoptosis in the presence of SPARC overexpression and irradiation.

\section{Discussion}

It is well established that the induction of apoptosis is critical to prevent the progression of any cancer. SPARC, through its inherent involvement in directing ECM deposition, cell-ECM interactions and growth factor signaling, plays numerous roles in regulating the multiple hallmarks of cancer including angiogenesis, migration, proliferation and survival (5). The results of the present study demonstrate that the combination treatment of
SPARC overexpression and irradiation induces apoptosis in a synergistic manner in neuroblastoma cells. However, the tumor suppressive properties of SPARC are highly dependent on various aspects, especially cell phenotype and the tumor microenvironment $(25,26)$. Hence, elucidation of the mechanisms underlying SPARC-mediated tumor suppression and the numerous confounding factors have been investigated in order to develop therapeutic strategies to confront cancer growth and metastasis. Activation of caspases is recognized as a critical event in most of the anticancer signaling pathways (27). SPARC overexpression alone or in combination with radiation treatment has been shown to enhance cleavage of caspase 3 and PARP in neuroblastoma cells. Further, our study demonstrates that SPARC overexpres- 
sion directed ER stress as a function of apoptosis via activation of ER stress transducers (e.g., PERK, IRE $1 \alpha$ and BiP).

The ER performs diverse functions including protein folding and also plays a major role in calcium homeostasis (28). It is well known that protein folding occurs in the ER prior to transport to various extracellular surface or intracellular organelles. There is significant evidence suggesting the essential role of ER in the regulation of apoptosis as well as autophagy $(16,29)$. Several types of cellular stress conditions can affect the protein folding process. Since unfolded or misfolded protein presents a threat to the cell, the ER lumen triggers the unfolded protein response (UPR) to circumvent cellular damage. The response to this ER stress induces activation of inositol-requiring endoplasmic reticulum-to-nucleus signal kinase (IRE)-1 $\alpha$ (30). In addition, RNA-dependent protein kinase (PKR)-like ER kinase (PERK) is a ubiquitous short-term perturbation to ER stress that leads to transduction of luminal signals across the ER membrane to its cytosolic kinase domain (31). This, in turn, changes the reserve ER chaperone leading to expression of molecules like $\mathrm{BiP}$. Given that BiP overexpression is known to suppress the UPR, enhanced expression of BiP contributes to effective ER stress response (32). Further, PERK amplification also leads to the phosphorylation of the $\alpha$ subunit of the translation factor, eIF $2 \alpha$, that ultimately inhibits protein synthesis by impeding the assembly of the 80 s ribosome (30). This scenario presents pertinent evidence for the involvement of ER stress in the signaling cascade. We observed the upregulation of PERK, IRE $1 \alpha$ and BiP in both SK-N-AS and NB-1691 cells at an earlier time-point after SPARC transfection. These ER stress transducers in turn induced activation of the transcription factor CHOP (Fig. 2D).

Likewise, multiple pathways seem to be involved in ER stress-initiated apoptosis, including the formation of autophagosomes and activation of JNK as illustrated by the expression of autophagy markers and active participation of kinases in regulating apoptosis. The diverse signaling activities associated with SPARC overexpression also highlight the ER protein flux. Even though the entire mechanism associated with ER stress-mediated apoptosis is unclear, the downstream ER stress signaling could be correlated with activation of $\mathrm{CHOP}$ (33). Further, it has been previously shown that activated IRE recruits the scaffolding protein TRAF2 to the ER membrane, triggering the mitogen-activated protein (MAP) kinase cascade and leading to c-Jun N-terminal kinase (JNK) activation (22).

It is further apparent from the results that when active JNK was inhibited after SPARC overexpression, the ER stress transducers were downregulated; this result exemplifies the involvement of ER molecular chaperones in eliciting c-Jun N-terminal kinase as part of the signaling cascade. It was found that when phosphorylation of JNK was inhibited, apoptosis was inhibited in both cell lines, which confirms the active involvement of JNK in regulating apoptosis. In addition, when phosphorylation of JNK was inhibited, the expressions of ER stress molecules PERK, BiP and IRE were also downregulated, thereby demonstrating that ER stress activation induces phosphorylation of JNK. Further, the role of ER stress in directing apoptosis was tested using an ER stress inhibitor. We found that when ER stress was inhibited, apoptosis was significantly reduced (Fig. 4), which further confirms the role of ER stress in the signaling cascade associated with SPARC overexpression leading to autophagy-mediated apoptosis in neuroblastoma cells.
Autophagy involves sequestration of autophagosomes that eventually fuse with lysosomes leading to cellular degradation and has an important role in eukaryotic cells. Autophagy is regulated by a set of evolutionarily conserved autophagyrelated (Atg) proteins. It was found that microtubule-associated protein light chain 3 II (LC3-II) is formed as a result of phosphoatidylethanolamine conjugation of LC3-I (also known as Atg8) indicate the formation of autophagosomes (34). Increased LC3 levels emphasize the coupling of UPR to autophagy in neuroblastoma. It has been previously demonstrated by the authors that SPARC overexpression leads to autophagy-mediated apoptosis in medulloblastoma (12). Even though the cross-talk between ER stress-induced autophagy and apoptosis is not completely understood, recent reports propose that PERK-eIF2 $\alpha$ pathway or IRE-TRAF2-JNK pathway could be the crucial mediator of ER stress-induced autophagy (15).

This study also provides strong evidence that integrating radiation as part of the combination treatment intensified the degree of apoptosis by sensitizing the cells in a synergistic manner as indicated by flow cytometry analysis, TUNEL assay, and activation of apoptotic molecules like caspase 3 and cleavage of PARP. Given the complexity of events initiated as part of ER stress induction, it could be proposed that there is an interplay of multiple intracellular pathways during SPARCinduced autophagy and apoptosis in neuroblastoma cells. The efficacy of the proposed hypothesis when tested in vivo showed concomitant results in the expression of ER stress molecular chaperons as a function of increased SPARC levels. These results confirm that ER stress has a key role in regulating the induction of apoptosis in SPARC-overexpressed neuroblastoma cells. The immunohistochemical analysis of phospho-JNK and LC3 expression levels in response to increased SPARC levels and the combination treatment further corroborates the in vitro results. In conclusion the results impart new insights regarding ER stress-mediated apoptosis in SPARC-overexpressed cells that should be explored further as a potential therapeutic option for neuroblastoma.

\section{Acknowledgements}

The authors thank Debbie McCollum for assistance in manuscript preparation, and Diana Meister and Sushma Jasti for manuscript review. We also thank Dr P. Houghton (St. Jude Children's Research Hospital, Memphis, TN) for providing the NB-1691 neuroblastoma cell line. This study was funded by NCI CA147792 to Jasti S. Rao from the National Institutes of Health (NIH).

\section{References}

1. Maris JM and Matthay KK: Molecular biology of neuroblastoma. J Clin Oncol 17: 2264-2279, 1999.

2. Smith MA, Seibel NL, Altekruse SF, et al: Outcomes for children and adolescents with cancer: challenges for the twenty-first century. J Clin Oncol 28: 2625-2634, 2010.

3. Jennings RW, LaQuaglia MP, Leong K, Hendren WH and Adzick NS: Fetal neuroblastoma: prenatal diagnosis and natural history. J Pediatr Surg 28: 1168-1174, 1993.

4. Fulda S and Debatin KM: Apoptosis pathways in neuroblastoma therapy. Cancer Lett 197: 131-135, 2003.

5. Arnold SA and Brekken RA: SPARC: a matricellular regulator of tumorigenesis. J Cell Commun Signal 3: 255-273, 2009. 
6. Mok SC, Chan WY, Wong KK, Muto MG and Berkowitz RS SPARC, an extracellular matrix protein with tumor-suppressing activity in human ovarian epithelial cells. Oncogene 12: 1895-1901, 1996.

7. Sato N, Fukushima N, Maehara N, et al: SPARC/osteonectin is a frequent target for aberrant methylation in pancreatic adenocarcinoma and a mediator of tumor-stromal interactions. Oncogene 22: 5021-5030, 2003.

8. Sova P, Feng Q, Geiss G, et al: Discovery of novel methylation biomarkers in cervical carcinoma by global demethylation and microarray analysis. Cancer Epidemiol Biomarkers Prev 15: 114-123, 2006

9. Chen Y, Chen JC and Tseng SH: Effects of tetrandrine plus radiation on neuroblastoma cells. Anticancer Res 29: 3163-3171, 2009.

10. Chen M, Hough AM and Lawrence TS: The role of p53 in gemcitabine-mediated cytotoxicity and radiosensitization. Cancer Chemother Pharmacol 45: 369-374, 2000.

11. Steel GG and Peckham MJ: Exploitable mechanisms in combined radiotherapy-chemotherapy: the concept of additivity. Int J Radia Oncol Biol Phys 5: 85-91, 1979.

12. Heck A: [Endoscopic operations spare the patient. Rapid rehabilitation, dispensing with large incisions, removal of appendix, gallbladder and esophagus]. Krankenpfl J 29: 148-149, 1991 (In German).

13. Bhoopathi P, Gondi CS, Gujrati M, Dinh DH and Lakka SS SPARC mediates Src-induced disruption of actin cytoskeleton via inactivation of small GTPases Rho-Rac-Cdc42. Cell Signal 23: 1978-1987, 2011.

14. Bhoopathi P, Chetty C, Gujrati M, Dinh DH, Rao JS and Lakka SS Cathepsin B facilitates autophagy mediated apoptosis in SPARC overexpressed primitive neuroectodermal tumor cells. Cell Death Differ 17: 1529-1539, 2010.

15. Hoyer-Hansen $\mathbf{M}$ and Jaattela M: Connecting endoplasmic reticulum stress to autophagy by unfolded protein response and calcium. Cell Death Differ 14: 1576-1582, 2007.

16. Ding WX, Ni HM, Gao W, et al: Differential effects of endoplasmic reticulum stress-induced autophagy on cell survival. J Biol Chem 282: 4702-4710, 2007.

17. Bhoopathi P, Chetty C, Gujrati M, Dinh DH, Rao JS and Lakka SS The role of MMP-9 in the anti-angiogenic effect of secreted protein acidic and rich in cysteine. Br J Cancer 102: 530-540, 2010.

18. Mohanam S, Jasti SL, Kondraganti SR, et al: Stable transfection of urokinase-type plasminogen activator antisense construct modulates invasion of human glioblastoma cells. Clin Cancer Res 7: 2519-2526, 2001.

19. Bhoopathi P, Chetty C, Kunigal S, Vanamala SK, Rao JS and Lakka SS: Blockade of tumor growth due to matrix metalloproteinase- 9 inhibition is mediated by sequential activation of beta1-integrin, ERK, and NF-kappaB. J Biol Chem 283: 1545-1552, 2008.
20. Chetty C, Lakka SS, Bhoopathi P, Kunigal S, Geiss R and Rao JS: Tissue inhibitor of metalloproteinase 3 suppresses tumor angiogenesis in matrix metalloproteinase 2-down-regulated lung cancer. Cancer Res 68: 4736-4745, 2008.

21. Tivnan A, Tracey L, Buckley PG, Alcock LC, Davidoff AM and Stallings RL: MicroRNA-34a is a potent tumor suppressor molecule in vivo in neuroblastoma. BMC Cancer 11: 33, 2011.

22. Ogata M, Hino S, Saito A, et al: Autophagy is activated for cell survival after endoplasmic reticulum stress. Mol Cell Biol 26: 9220-9231, 2006.

23. Liu J and Lin A: Role of JNK activation in apoptosis: a doubleedged sword. Cell Res 15: 36-42, 2005.

24. Hetz C: The unfolded protein response: controlling cell fate decisions under ER stress and beyond. Nat Rev Mol Cell Biol 13: 89-102, 2012

25. Clark CJ and Sage EH: A prototypic matricellular protein in the tumor microenvironment - where there's SPARC, there's fire. J Cell Biochem 104: 721-732, 2008.

26. Podhajcer OL, Benedetti L, Girotti MR, Prada F, Salvatierra E and Llera AS: The role of the matricellular protein SPARC in the dynamic interaction between the tumor and the host. Cancer Metastasis Rev 27: 691-705, 2008.

27. Fulda S and Debatin KM: IFNgamma sensitizes for apoptosis by upregulating caspase- 8 expression through the Stat1 pathway. Oncogene 21: 2295-2308, 2002.

28. Deniaud A, Sharaf el Dein O, Maillier E, Poncet D, Kroemer G, Lemaire $\mathrm{C}$ and Brenner C: Endoplasmic reticulum stress induces calcium-dependent permeability transition, mitochondrial outer membrane permeabilization and apoptosis. Oncogene 27: 285-299, 2008.

29. Kuma A, Hatano M, Matsui M, et al: The role of autophagy during the early neonatal starvation period. Nature 432: 10321036,2004

30. Urano F, Wang X, Bertolotti A, Zhang Y, Chung P, Harding HP and Ron D: Coupling of stress in the ER to activation of JNK protein kinases by transmembrane protein kinase IRE1. Science 287: 664-666, 2000

31. Lin JH, Li H, Zhang Y, Ron D and Walter P: Divergent effects of PERK and IRE1 signaling on cell viability. PLoS One 4: e4170, 2009.

32. Bertolotti A, Zhang Y, Hendershot LM, Harding HP and Ron D: Dynamic interaction of $\mathrm{BiP}$ and ER stress transducers in the unfolded-protein response. Nat Cell Biol 2: 326-332, 2000.

33. Zinszner H, Kuroda M, Wang X, et al: CHOP is implicated in programmed cell death in response to impaired function of the endoplasmic reticulum. Genes Dev 12: 982-995, 1998.

34. Mah LY and Ryan KM: Autophagy and cancer. Cold Spring Harb Perspect Biol 4: a008821, 2012. 